\title{
Review of: "Feature-based sequencing optimization method for minimizing non-cutting energy consumption for CNC machine tools"
}

Jun Zheng

Potential competing interests: The author(s) declared that no potential competing interests exist.

1. Please make sure to clearly demonstrate the relevance and advantage of your study to energy consumption in the introduction.

2. The text should be meticulously checked for accuracy and readability.

3.What's the advantages of this research? What's the areas for further improvement?

4.The wording problem in the paper: i feature and feature i appear several times in the article $\square$ and the author should use a uniform format (j feature also); change step one to step 1 on page 7.

5.Problems with the charts in the paper: the elements in the cutting parameters column in Table 1 are not aligned; the specific time units in Table 4 are not indicated, and there are no specific units in Table 5 and Table 7; the format of Table 3 is a bit messy, and the author should recreate Table 3.

5 Problems with the equations in the paper: "which is

$$
t_{\max }=\max \left(t_{X}, t_{Y}, t_{Z}\right)
$$

" below equation (5) should be changed to "which is

$$
t_{\max }=\max \left(t_{X F}, t_{Y F}, t_{Z F}\right)
$$

"; Equation (10) equation (13) should be aligned;

$$
E_{c t}^{(F i-F j)}
$$

below equation (14) should be changed to

$$
E_{c t}^{(F i, F j)}
$$

; Is

$$
k_{1}
$$


the same in equation (7) as it is in equation (10)? Is

\section{$b_{2}$}

the same in equation (7) as it is in equation (11)?

7.The authors should explain the origin of the specific constant values in the equations of Table 3.

8.The authors should explain the origin of the ordering of the other features in Table 8.

9. In the conclusion section, summarize the main points of the article, as well as your opinions about its significance, accuracy, and clarity. If relevant, also comment on implications for further research or discussion in the field. 